\title{
Fator de Aumento de Dose em Radioterapia com Nanopartículas: Estudo por simulação Monte Carlo
}

\author{
Dose Enhancement Factor in Radiation Therapy with nanoparticles: \\ A Monte Carlo-Simulation Study
}

\author{
Vinícius F. Santos ${ }^{1}$, Patrícia Nicolucci ${ }^{1}$ \\ ${ }^{1}$ Universidade de São Paulo, Faculdade de Filosofia Ciências e Letras de Ribeirão Preto \\ Departamento de Física, Ribeirão Preto, Brasil
}

\begin{abstract}
Resumo
A incorporação de nanopartículas metálicas em tecidos tumorais tem sido estudada em Radioterapia devido ao aumento de dose que pode ser obtido no volume alvo do tratamento. Estudos indicam que nanopartículas de ouro estão entre as de maior viabilidade biológica para essas aplicações, devido ao baixo potencial tóxico em comparação com outros metais. Além disso, estudos mostram que nanopartículas de alguns poucos nanômetros até alguns micrômetros podem permear vasos sanguíneos que alimentam tumores, permitindo sua incorporação nas células tumorais. A internalização de nanopartículas de ouro em células tumorais associada ao uso de feixes clínicos de energias apropriadas pode, desta forma, proporcionar não apenas fatores de aumento de dose, mas podem, também, proporcionar a radiossensibilização das células tumorais, aumentando o controle tumoral. Desta forma, este trabalho visa estudar o fator de aumento de dose obtido em Radioterapia com nanopartículas de ouro incorporadas ao tecido tumoral utilizando feixes de ortovoltagem, de braquiterapia e de teleterapia. Para tanto, foi utilizado neste trabalho uma metodologia computacional, através de simulação Monte Carlo, com o código PENELOPE, simulando feixes clínicos de 50 e $150 \mathrm{kVp},{ }^{192} \mathrm{Ir}$ e $6 \mathrm{MV}$, e um modelo de célula tumoral com nanopartículas de ouro incorporadas. Foram utilizadas diferentes concentrações de nanopartículas de ouro, calculadas por fração em massa, preenchendo o núcleo da célula, de $2 \mu \mathrm{m}$ de diâmetro, de forma homogênea. Os resultados mostraram um fator de aumento de dose significativo para feixes de ortovoltagem com maiores concentrações de nanopartículas de ouro, como para 50 e $150 \mathrm{kVp}$. Para esses feixes, os maiores fatores de aumento de dose encontrados foram de, respectivamente, 1,61 $\pm 0,09$ e $2,76 \pm 0,09$. Os resultados obtidos neste estudo podem ser utilizados como ponto de partida para estudo de radiossensibilização utilizando nanopartículas em um modelo de distribuição heterogênea utilizando-se as energias e concentrações que otimizem a resposta do tumor.
\end{abstract}

Palavras-chave: Radioterapia, nanopartículas de ouro, fator de aumento de dose, simulação Monte Carlo.

\section{Abstract}

The incorporation of metallic nanoparticles into tumor tissues has been studied in radiation therapy due to the increase of dose that can be obtained in the target volume of the treatment. Studies indicate that gold nanoparticles are among the most biologically viable for these applications because of the low toxic potential compared to other metals. In addition, studies show that nanoparticles from a few nanometers to a few micrometers can permeate blood vessels that feed tumors, allowing their incorporation into tumor cells. The internalization of gold nanoparticles in tumor cells associated with the use of clinical beams of appropriate energies may, therefore, not only provide dose enhancement factors, but may also provide the radiosensitization of tumor cells, increasing the tumor control. Hence, this work aims to study the dose enhancement factor obtained in Radiation Therapy with gold nanoparticles incorporated into the tumor tissue using orthovoltage, brachytherapy and teletherapy beams. Monte Carlo simulations with the PENELOPE package, were used to simulate 50 and $150 \mathrm{kVp},{ }^{192} \mathrm{Ir}$ and $6 \mathrm{MV}$ clinical beams, and a tumor cell model with gold nanoparticles incorporated. Different concentrations of nanoparticles, calculated by mass fraction, were homogeneously simulated in the cell nucleus of $2 \mu m$-diameter. The results presented show a significant dose enhancement factor for the orthovoltage energies with higher concentrations of gold nanoparticles, such as for 50 and $150 \mathrm{kVp}$. For these beams, the higher dose enhancement factors found were 1,61 $\pm 0,09$ and 2,76 $\pm 0,09$, respectively. The results of this study can be used as a starting point for the study of radiossensitization using nanoparticles with a heterogeneous model for the nanoparticles distribution inside the cells using the beams and concentrations that optimize the effect on the tumor.

Keywords: Radiation Therapy, gold nanoparticle, dose enhancement factor, Monte Carlo simulation. 


\section{Introdução}

A incorporação de nanopartículas metálicas em tecidos tumorais tem sido estudada em Radioterapia devido ao aumento de dose que pode ser obtido no volume alvo do tratamento, que ocorre pelas várias interações físicas que acontecem, quando se tem uma energia baixa interagindo com o material de alto número atômico (Z) no meio ${ }^{1,2}$. Dado que os feixes clínicos mais utilizados em radioterapia são de megavoltagem, diversos estudos da internalização de nanopartículas de ouro (AuNPs) em células tumorais tem sido realizados nessas energias ${ }^{3.4}$. Entretanto, a associação de AuNPs e feixes de baixas energias pode proporcionar um aumento potencial na resposta tumoral, conseqüente do aumento na interação entre o tecido e a radiação, principalmente através do efeito fotoelétrico ${ }^{5-7}$.

A literatura mostra que AuNPs de alguns nanômetros apresentam baixa toxicidade e podem permear vasos sanguíneos que alimentam tumores, proporcionando um efeito de radiossensibilização diferencial nesses tecidos em relação aos tecidos normais ${ }^{4}$. Nesse sentido, o uso de feixes de radiação de energias como as utilizadas em ortovoltagem e braquiterapia poderia trazer uma vantagem potencial para radioterapia com nanopartículas em relação aos feixes de teleterapia, que têm energias relativamente mais elevadas ${ }^{8}$.

Desta forma, o objetivo deste trabalho é investigar o fator de aumento de dose obtido com diferentes concentrações de AuNPs homogeneamente incorporadas no nucléolo da célula para feixes clínicos de diferentes energias.

\section{Materiais e Métodos}

O presente trabalho foi realizado através de uma metodologia computacional, com simulação Monte Carlo, com o pacote PENELOPE, para o estudo do fator de aumento de dose em Radioterapia utilizando AuNPs incorporadas ao volume tumoral.

\subsection{Simulação Monte Carlo PENELOPE}

O pacote de simulação Monte Carlo PENELOPE (PENetration and Energy LOss of Positrons and Electrons) pode ser utilizado para simular o transporte de elétrons, pósitrons e fótons em geometrias complexas ${ }^{9}$.

Os parâmetros de entrada das simulações realizadas foram definidos de modo a otimizar o desempenho das simulações. Para tanto, foram utilizadas $10^{8}$ partículas primárias para cada simulação. As energias de corte para fótons e partículas carregadas foram definidas em $10 \mathrm{keV}$ para todas as energias de feixes utilizadas. Os parâmetros C1 e C2, que controlam a condensação de histórias de partículas carregadas secundárias, foram mantidos em 0,05 em todas as simulações, de modo a garantir exatidão nas doses simuladas no entorno das AuNPs.

\subsection{Geometria de simulação}

O pacote PENELOPE foi utilizado para geração de arquivos de materiais compostos por uma mistura homogênea de água e ouro, em concentrações de 0,$01 ; 0,05 ; 0,1 ; 0,5 ; 1,0 ; 1,1 \%$ de massa. O código PENGEOM, do pacote PENELOPE, foi utilizado para representação de um modelo de célula ${ }^{10}$, apresentada na Figura 1, com diferentes tamanhos para as seguintes estruturas: membrana celular, retículo endoplasmático, núcleo e nucléolo com os tamanhos de 13, 6, 3 e $2 \mu \mathrm{m}$ de diâmetro, respectivamente. Nas simulações, somente o nucléolo foi preenchido com o material formado pela mistura de água mais ouro, nas diversas concentrações estudadas, e o restante das estruturas foram preenchidas com água.

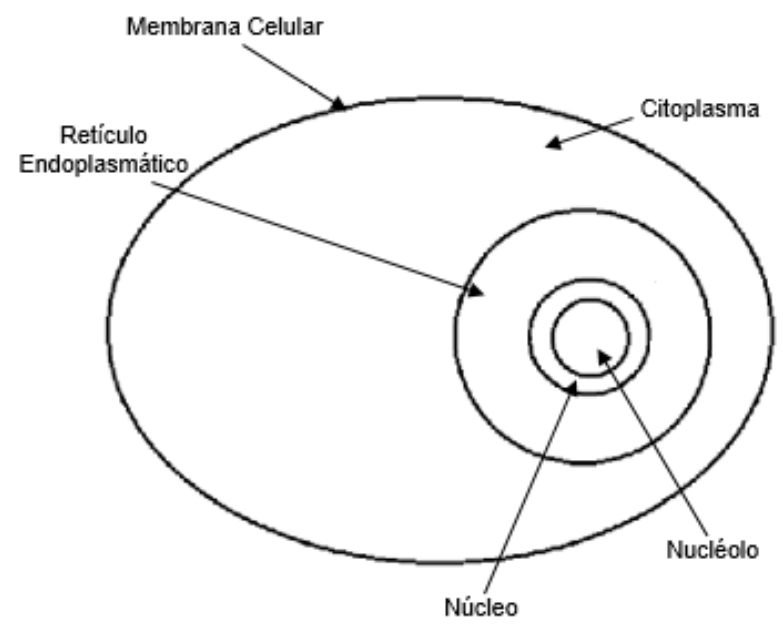

Figura 1: Modelo de célula utilizado nas simulações (Adaptado de Douglass et. al, 2012) $^{10}$.

\subsection{Fator de aumento de dose}

O fator de aumento de dose (DEF, do inglês Dose Enhancement Factor) é definido como a razão entre a dose total entregue no volume com nanopartículas incorporadas e a dose total integrada no mesmo volume sem a presença das nanopartículas, sendo dado pela equação 1 :

$$
D E F=\left[\frac{D_{\text {com AunPs }}}{D_{\text {sem AunPs }}}\right]
$$

O DEF foi avaliado por um modelo de incorporação homogênea de ouro no tecido, sem que a interface da nanoestrutura do material seja considerada. Desta forma, o aumento de dose é considerado como consequência do aumento no número atômico efetivo do material incorporado. As doses totais no volume da célula preenchida com e sem AuNP foram comparadas em função da 
concentração de ouro e da energia dos feixes utilizados.

\subsection{Espectros dos feixes estudados}

No estudo foram utilizados espectros de feixes clínicos de ortovoltagem, de 50 e $150 \mathrm{kVp}$, fonte de braquiterapia com ${ }^{192}$ Ir e também de um acelerador linear de $6 \mathrm{MV}^{11-15}$, conforme mostrados na Figura 2.

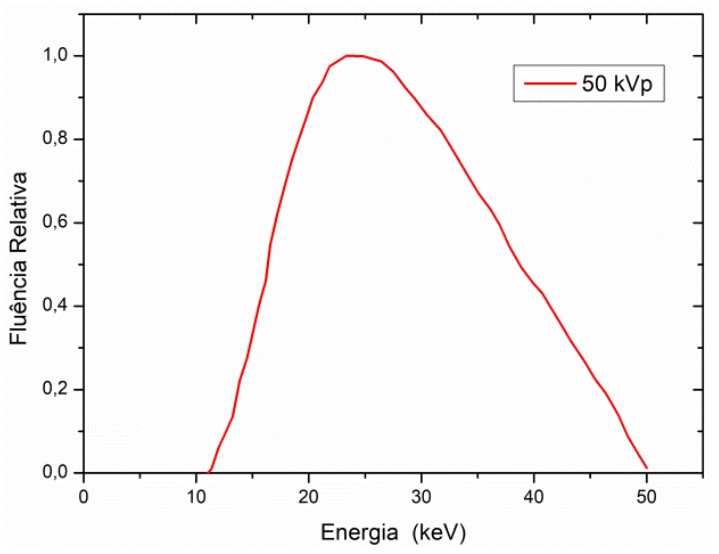

(a) (Adaptado de Rosenschold, et. al., 2008)

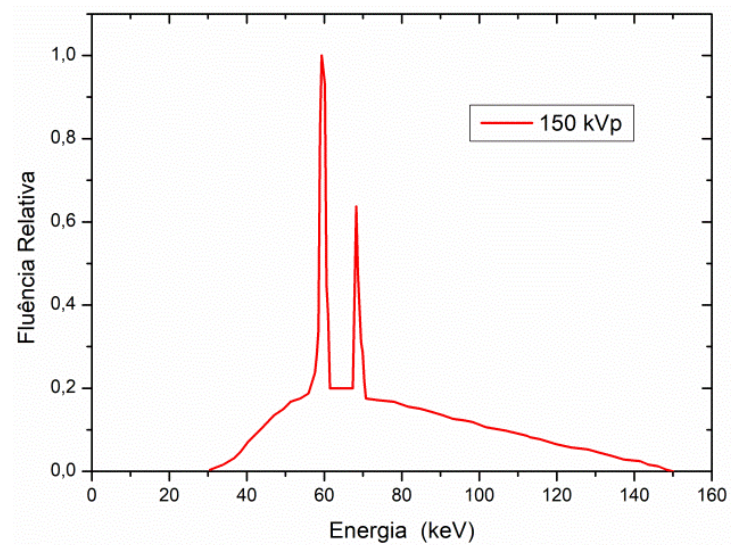

(b) (Adaptado de Brown, S. et al., 2011)

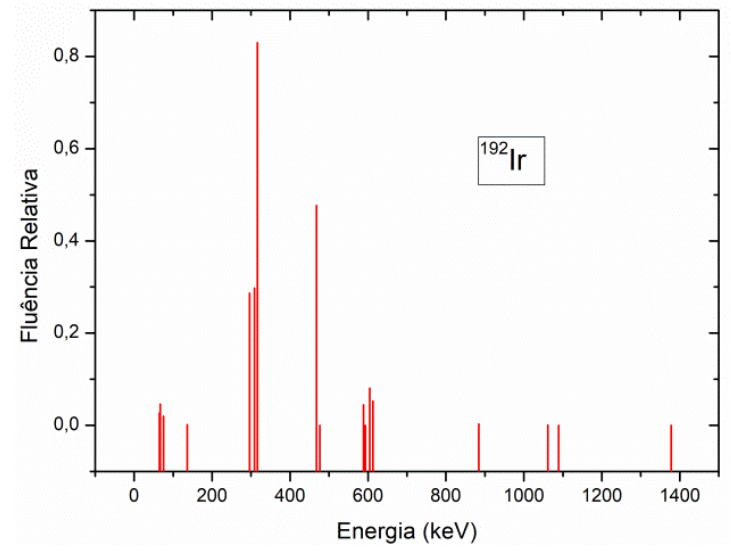

(c) (Adaptado de Zilio, V. O. et al., 2004)

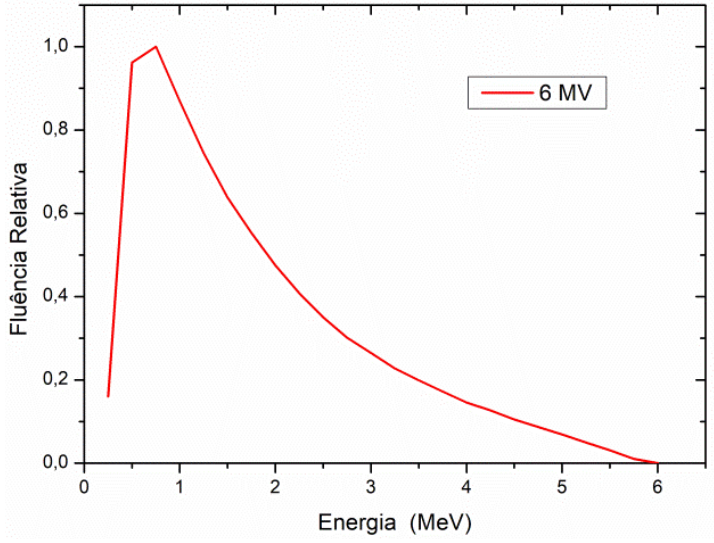

(d) (Adaptado de Sheikh-Bagheri, D. e Rogers, D. W. O., 2002)

Figura 2: Espectros de energia dos feixes clínicos de $50 \mathrm{kVp}$ (a), 150 kVp (b), Ir-192 (c) e 6 MV (d) utilizados no trabalho.

\section{Resultados e Discussão}

Através das simulações utilizando a geometria da célula foram obtidos os DEFs no modelo de incorporação homogênea de AuNPs para os quatro feixes clínicos utilizados e com as diferentes concentrações. Os DEFs para essas diferentes combinações de concentração e feixes são mostrados na Figura 3.

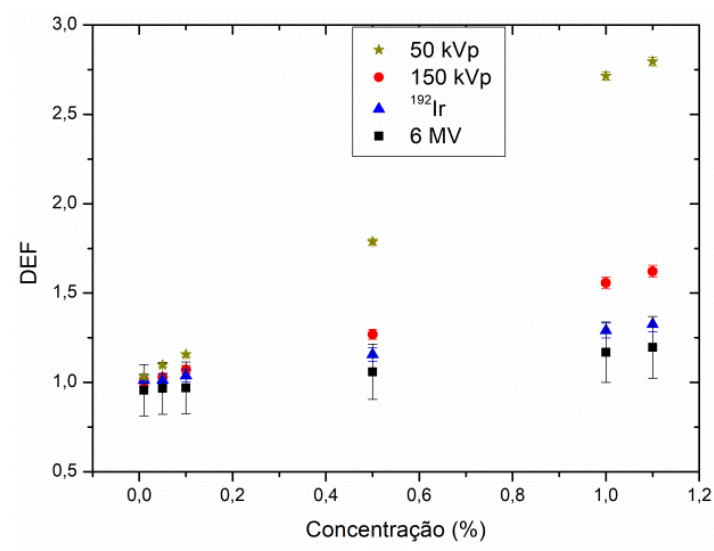

Figura 3. Fatores de aumento de dose em função das concentrações de AuNPs e feixes clínicos.

Conforme esperado, as maiores concentrações de AuNPs incorporadas no volume produzem maiores DEFs, devido ao aumento no número atômico efetivo do material e, consequentemente, maior interação e absorção da radiação. Além disso, de modo geral, os feixes de menor energia produzem maior DEF que os de maior energia, devido ao aumento das interações da radiação de baixas energias com materiais de alto número atômico.

$\mathrm{Na}$ Figura 3 pode-se notar, ainda, como padrão geral, as maiores incertezas associadas aos DEFs obtidos para o feixe de $6 \mathrm{MV}$. Isto ocorreu devido ao uso dos mesmos parâmetros de simulação em todas as simulações. A menor probabilidade de 
interação de feixes de megavoltagem, em comparação com os feixes de kilovoltagem, exige a utilização de um número de partículas primárias maior para que se obtenha a mesma incerteza associada a essas simulações. Neste trabalho, entretanto, o uso dos mesmos parâmetros de simulação, para todas as condições estudadas, foi escolhido para privilegiar a comparação das diferentes doses obtidas em cada combinação de concentração e feixe.

Os DEFs, para cada energia em diferentes concentrações, são representados na Tabela 1. O aumento significativo do DEF para os feixes de ortovoltagem é devido à maior contribuição de efeito fotoelétrico entre esses feixes e o ouro, aumentando a fluência de elétrons secundários de baixas energias e, por conseguinte, a dose local.

Tabela 1.Fatores de aumento de dose para todas energias estudadas em diferentes concentrações

\begin{tabular}{ccccccc}
\hline \multicolumn{7}{c}{ Concentração (\%massa) } \\
\hline Feixe & $\mathbf{0 , 0 1}$ & $\mathbf{0 , 0 5}$ & $\mathbf{0 , 1}$ & $\mathbf{0 , 5}$ & $\mathbf{1 , 0}$ & $\mathbf{1 , 1}$ \\
\hline $\mathbf{5 0} \mathbf{~ k V p}$ & 1,02 & 1,10 & 1,12 & 1,72 & 2,59 & 2,77 \\
$\mathbf{1 5 0 ~ k V p}$ & 1,00 & 1,02 & 1,04 & 1,26 & 1,55 & 1,62 \\
${ }_{\mathbf{1 9 2}} \mathbf{~ r}$ & 0,99 & 1,00 & 1,02 & 1,14 & 1,29 & 1,31 \\
$\mathbf{6} \mathbf{~ M V}$ & 0,97 & 0,98 & 1,00 & 1,09 & 1,20 & 1,22 \\
\hline
\end{tabular}

Para os feixes de ${ }^{192}$ Ir e $6 \mathrm{MV}$, com menores concentrações, os DEFs menores que 1 encontrados são devidos às incertezas estatísticas obtidas nas simulações. A diminuição na dose no tecido biológico, entretanto, pode ser um problema real em situações de incorporação de AuNPs, dado a autoatenuação da radiação secundária criada nas nanopartículas e a diminuição correspondente de fluência de radiação secundária no material biológico.

Embora a grande maioria dos trabalhos da literatura estudem o uso de AuNPs em radioterapia de megavoltagem, os resultados obtidos neste trabalho mostram que a maior potencialidade de uso de radioterapia com AuNPs pode ser dada com o uso de feixes de ortovoltagem. Enquanto que a incorporação de AuNPs em tumores profundos encontra grande dificuldade no desenvolvimento de agentes carreadores específicos, os tumores de pele, por exemplo, que podem ser tratados com feixes de ortovoltagem, podem ser expostos às nanopartículas por meio de géis tópicos, que permitem que apenas o tumor incorpore as AuNPs, protegendo os tecidos normais adjacentes do aumento de dose. Além disso, um aumento de dose similar poderia ser obtido com o uso de menores concentrações de nanopartículas, diminuindo potenciais efeitos tóxicos ${ }^{16}$. Desta forma, o uso de radioterapia com AuNPs para tumores superficiais poderia se beneficiar das sinergias entre os maiores DEFs e a maior especificidade do agente carreador das nanopartículas.

Os DEFs obtidos neste trabalho, para diferentes feixes clínicos e concentrações de AuNPs, podem ser utilizados como ponto de partida para escolha de parâmetros de estudo em modelos mais realísticos de radioterapia com nanopartículas, sendo que estes resultados corroboram com estudo de Mesbahi e colaboradores que apresentam DEFs próximos aos encontrados neste trabalho também para energias de ortovoltagem, variando entre 1,8 e 3,3 para $50 \mathrm{keV}$, por exemplo, enquanto que a encontrada neste estudo para a mesma energia foi de 1,02 à 2,77, resultados que confirmar a eficácia da combinação de um feixe de baixa energia com uso de AuNPs ${ }^{17}$. Podemos citar ainda outros estudos como, por exemplo, Brivio e colaboradores, que utilizam de AuNPs em radiocirurgia de mácula em combinação com feixe de kilovoltagem de $100 \mathrm{kVp}$, obtendo resultados satisfatórios e que demonstram a eficácia da utilização das AuNPs para tal aplicação, apresentando um aumento na razão de aumento de dose (DER) de 1,97 $\pm 0,03$ direto na célula macular endotelial $^{18}$. Outro estudo de referência na utilização de AuNPs é para o estudo de Anijdan e colaboradores, onde eles analisam a influência de AuNPs no tratamento de tumores em ratos com feixes de megavoltagem, realizando uma análise entre o tratamento, tratamento apenas com aplicação de AuNPs, tratamento com irradiação e ainda AuNP mais irradiação combinados. A combinação de AuNPs e irradiação demonstram um resultado satisfatório na sobrevivência dos ratos, obtendo um DEF entre 8 e $10 \%$ na análise após as irradiações ${ }^{19}$. Em nossos estudos o feixe de megavoltagem foi o que representou o menor valor de DEF, por outro lado, para outros estudos podemos observar que é possível obter aumento no tratamento com megavoltagem significante, demonstrando que tal feixe pode ser analisado com maior detalhe posteriormente nos próximos trabalhos, podendo representar um aumento de dose em maiores profundidades do tecido, sendo essa uma característica para tal feixe.

Para tanto, modelos de incorporação heterogênea das AuNPs no volume tumoral devem ser realizados para avaliação mais detalhada dos efeitos locais do aumento de dose no tumor.

\section{Conclusões}

O aumento de dose no tumor, proporcionado pela incorporação de AuNPs, é significativamente maior para feixes de ortovoltagem que para feixes convencionais de teleterapia com megavoltagem. Com base nos resultado apresentados neste trabalho pode-se concluir que feixes de ortovoltagem são os de maior potencialidade de aplicação em radioterapia com AuNPs, pois apresentam um fator de aumento de dose significativamente maior que feixes de megavoltagem, em todas concentrações estudadas.

\section{Referências}

1. Cho S H.: Estimation of tumor dose enhancement due to gold nanoparticles during typical radiation treatments: A preliminary Monte Carlo study. Med. Phys. v. 32, p.2162-2162, 2005.

2. Chithrani D B, Jelveh S, Jalali F, et al:: Gold nanoparticles as radiation sensitizers in cancer therpy. Rad. Research. v. 173, p. 719-728, 2010.

3. Wolfe T, Chatterjee D, Lee J, Grant JD, Bhattarai S, Tailor R, et al:: Targeted gold nanoparticles enhance sensitization of 
prostate tumors to megavoltage radiation therapy in vivo. Nanomed. v. 11, p. 1277-83, 2015.

4. Lakshmanan, V.: Therapeutic efficacy of nanomedicines for prostate cancer: An update. I. C. Urology. v. 57, p. 21-29, 2016.

5. Jones B L, Krishnan S, Cho S H.: Estimation of microscopic dose enhancement fator around gold nanoparticle by Monte Carlo calculations. Med. Phys. 37, 3809 (2010).

6. Albanese A, Tang P S, Chan W C W. The effect of nanoparticle size, shape, and surface chemistry on biological system. Annu. Ver. Biomed. Eng. v.14, p.1-16 (2012).

7. Lechtman E, Chattopadhyay N, Cai Z, et al.: Implications on clinical scenario of gold nanoparticle radiosensitization in regards to photon energy, nanoparticle size, concentration and location. Phys. Med. Biol. v. 56, p. 4631-4647. 2011.

8. Khoshgard K, Hashemi B , Arbabi A, et al:: Radiosensitization effect of folate-conjugated gold nanoparticles on HeLa cancer cells under orthovoltage superficial radiotherapy techniques. Phys. Med. Biol. v.59, p. 2249-2263. 2014.

9. Salvat F, Fernandez-Varea J M, Sempau J. PENELOPE-2008: A Code System for Monte Carlo Simulation of Electron and Photon Transport, Nuclear energy agency, Espanha, 2008.

10. Douglass M, Bezak E e Penfold S: Monte Carlo investigation of the increased radiation deposition due to gold nanoparticles using kilovoltage and megavoltage photons in a 3D randomized cell model. Med. Phys. v. 40, p. 071710.1$071710.9,2013$.

11. Rosenschold $M$, Nilsson $P$, Knoos $T$ : Kilovoltage $x$-ray dosimetry-an experimental comparison between different dosimetry protocols. Phys. Med. Biol. v. 53, p. 4431-4442, 2008.

12. Brown S, Detwiler R S, Lu B, et al.: Utilization of MCNP for the development of spectral stripping algorithms for orthovoltage radiation therapy systems. Nucl. Instr. Meth. Phys. Res. A. v. 652, p. 726-730, 2011.

13. Sheikh-Bagheri D e Rogers D W O: Monte Carlo calculation of nine megavoltage photn beam spectra using the BEAM code. Med. Phys. v. 29, p. 391-402, 2002.

14. Verhaegen F, Nahum A E, Putte S, et al.: Monte Carlo modelling of radiotherapy kV x-ray units. Phys. Med. Biol. v. 44, p. 1767-1789, 1999

15. Zilio V L, Joneja O P, Popowski $\mathrm{Y}$, et al.: Dosimetric Characterization of Radioactive Sources Employed in Prostate Cancer Therapy. Brachyther., v. 3, p. 201-214, 2004.

16. Alkilany A M, Murphy C J. Toxicity and cellular uptake of gold nanoparticle: what have we learned so far? J. Nanopart. Res. v.12, p.2313-2333, 2010.

17. Mesbahi A, et al:: Effect of Photon Beam Energy, Gold Nanoparticle Size and Concentration on Dose Enhancement in Radiation Therapy. Biolmpacts. v. 3, p. 29-35, 2013.

18. Brivio $\mathrm{D}$, et al.: Kilovoltage radiosurgery with gold nanoparticle for neovascular age-related macular degeneration (AMD): a Monte Carlo evaluation. Phys. Med. Biol. v. 60, p. 9203-9213, 2015.

19. Anijdan S H M, et al:: Megavoltage X-ray Dose Enhancement with Gold Nanoparticles in Tumor Bearing Mice. Int $\mathbf{J}$ Mol Cell Med. v. 2, p. 118-124, 2013.

\section{Contato:}

Vinícius Fernando dos Santos

Departamento de Física - FFCLRP

Avenida Bandeirantes, 3900 - Ribeirão Preto-SP

CEP 14040-901.

Universidade de São Paulo

e-mail: viniciusf_santos@hotmail.com 